Revista Internacional de Apoyo a la Inclusión, Logopedia, Sociedad y Multiculturalidad. Volumen 4, Número 2, Abril 2018, ISSN: 2387-0907, Dep. Legal: J-67-2015 http://riai.jimdo.com/

\title{
Educación continua para el equipo de enfermería sobre Calendário Nacional de Vacunación, Município de Santa Maria do Pará.
}

(Continuing education for the nursing team on National Vaccination Calendar, Municipality of Santa Maria do Pará.)

Elane Silva Costa

Escola Técnica do SUS-ETSUS, Santa Maria do Pará, Pará-Brasil

Fecha recepción: 11-01-2017

Páginas 70-84

Fecha aceptación: 30-03-2017

\section{Resumen.}

Es pertinente destacar la importancia de las vacunas a lo largo de sus 200 años, que contribuyeron a la reducción de la mortalidad de las enfermedades prevenibles inmunológicamente en la población. El objetivo de la investigación es Desarrollar un Programa de Educación Continua sobre el Calendario Nacional de Vacunación para el equipo de enfermería en el municipio de Santa María del Pará, Brasil. Evaluar un Instrumento didáctico actualizado para facilitar la práctica profesional e identificar las competencias y habilidades del equipo de enfermería propias en la sala de vacunación. Los resultados fueron procesados en el SPSS STATISTICS 18 con tablas y gráficos. Todavía, queda evidente en esta investigación la importancia de los cursos de Educación Continua sobre el Calendario Nacional de vacunación para el Equipo de Enfermería para que sea alcanzada la finalidad por el Ministerio de Salud identificando una mejora dela práctica profesional en las salas de vacunación.

Palabras claves: educación; continua; equipo; calendario; vacunación

\begin{abstract}
.
It is pertinent to highlight the importance of vaccines throughout their 200 years, which contributed to the reduction of mortality of immunologically preventable diseases in the population. The objective of the research is to develop a Continuing Education Program on the National Vaccination Calendar for the nursing team in the municipality of Santa María del Pará, Brazil. Evaluate an updated didactic instrument to facilitate professional practice and identify the skills and abilities of the nursing team in the vaccination room. The results were processed in the SPSS STATISTICS 18 with tables and graphs. Still, it is evident in this investigation the importance of Continuing Education courses on the National Vaccination Calendar for the Nursing Team so that the Ministry of Health can reach the finality, identifying an improvement of the professional practice in the vaccination rooms.
\end{abstract}

Keywords: education; keep going; equipment; calendar; vaccination 


\section{1.-Introducción.}

A lo largo de 200 años las vacunas vienen contribuyendo en la reducción de la morbimortalidad de las enfermedades "inmunoprevenibles" en la población, con esto se mostró su importancia a Nivel Mundial, Nacional, Estadual y Municipal. Para Cavalcante (2008), en el siglo XX, Oswaldo Cruz emprende en Brasil una obra sanadora que se convertiría en uno de los episodios más convenientes de la historia de la salud pública mundial. El destacado sanitarista establece un modelo de acción y dejó un ejemplo que sirvió de inspiración para el Programa Nacional de Inmunización (PNI), que es hoy citado como referencia mundial por los avances notables y su excelencia comprobada.

Según relata este autor, desde las primeras vacunaciones en 1804, Brasil acumuló más de 200 años de experiencia en inmunizaciones y con la implantación del PNI en los últimos 40 años desarrollaron acciones planeadas y sistematizadas, consiguiendo erradicar algunas enfermedades. Como podemos observar, el Programa Nacional de Inmunizaciones avanza en el sentido de posibilitar a la población brasileña la protección contra enfermedades perfectamente prevenibles por vacunas que tienen influencia directa en la reducción de los cuadros de morbimortalidad.

Las vacunas son usadas desde la $2^{\mathrm{a}}$ Guerra Mundial y fueron suficientemente eficaces para reducir drásticamente la morbilidad y mortalidad causada por varias enfermedades, brindando credibilidad a los programas de prevención contra enfermedades infecciosas. La explosión científica que ocurrió en aquel período, originó la nuevas vacunas, algunas de las cuales aún se utilizan como es el caso de la Polio, Sarampión, Parotiditis y Rubéola (Feliciano, 2002). Vale destacar que en 1888 es fundado el Instituto Pasteur, centro de investigación biológica, principalmente en lucha contra las Enfermedades Infecciosas. Gracias a las escuelas francesas y alemán, la vacunación registra progresos notables, obteniendo vacunas contra la Fiebre Tifoidea, el Cólera, la Tos Convulsa, la Fiebre-amarilla y la Tuberculosis.

Las vacunas van más allá de la protección ofrecida a nivel individual, hay objetivos más amplios y de mayor alcance en la salud pública y tienen aplicabilidad en programas o campañas de vacunación bien planeadas y correctamente ejecutadas, y logran en una primera fase, el control de las enfermedades contagiosas entre los seres humanos de una comunidad y posteriormente tiene como objetivo final su erradicación. Se sabe que el avance tecnológico del Calendario Básico de Vacunación está en constante expansión, siendo necesario agregar nuevos conocimientos, para el buen desempeño del Equipo de Enfermería que actúa directamente en la Atención Primaria, responsables de la lectura del cartón de vacunación, indicación y aplicación de las Vacunas establecidas en el Calendario Nacional de Vacunación.

Es necesario invertir en Cursos de actualización para que los profesionales adquieran más conocimientos y seguridad en las salas de vacunación. Ciertamente, 
dicha calificación exige un cambio en el modelo de cuidado y prácticas de salud. La Comisión Permanente de Educación Continua es el proceso de trabajo del equipo de salud considerada dimensión lógica y nueva, rompiendo las viejas estructuras de poder entre estos profesionales de la salud y el público (Ministério de Salud, 2014).

El Equipo de Enfermería (Enfermeros y Técnicos de Enfermería), que actúa en las salas de vacunación para el control de enfermedades inmunoprevenibles, requiere conocimiento, dominio y seguridad al realizar sus actividades, entre las cuales destacamos: Organización de la Cadena de Frio, Organización de la Sala de Vacunación, administración de Inmunobiologicos, actualizaciones del cartón de vacunación, orientación sobre los efectos adversos por vacunación y de precisar, encaminar al Centro de Referencia para Inmunobiologicos Especiales (CRIE), para tal, el equipo debe estar bien entrenado y capacitado en esos temas, quedando evidente la importancia de la Educación Continua.

EL Problema del estudio consiste en que hay existe una deficiencia en el conocimiento por el Equipo de Enfermería relacionada con las nuevas vacunas que están siendo introducidas en el Calendario Nacional de Vacunación, debido al proceso de ampliación del mismo y a las innovaciones tecnológicas. Como se puede observar después del recibimiento de los egresos de las carreras de Técnico de Enfermería y en el caso de los Enfermeros, debido a la intensa carga de trabajo, muchas veces no tienen tiempo para que realicen cursos de actualización de Educación Continua fuera del municipio, entonces nos preguntamos: ¿ ¿Será que la Educación Continua serviría para actualizar el conocimiento sobre el Calendario Nacional de Vacunación actual para el Equipo de Enfermería en las unidades de salud de Santa María do Pará?

Se justifica por destacar la importancia de las vacunas para el control de las enfermedades prevenibles en la población. Actualmente, existe una deficiencia de conocimiento por el Equipo de Enfermería relacionada a las nuevas vacunas que están siendo introducidas en el Calendario Nacional de Vacunación del Ministerio de la Salud, debido al constante proceso de cambio y a las innovaciones tecnológicas.

El Equipo de Enfermería es parte de los profesionales que trabajan directamente con las vacunas y ellos son los encargados por el cumplimento del Calendario Nacional de Vacunación. Es necesario que el Equipo de Enfermería este inmerso en cursos de actualización de Educación Continua. En esta investigación pretendemos contribuir con la actualización del conocimiento de los profesionales del Equipo de Enfermería que actúan en las salas de vacunación. Teniendo como punto de partida la implementación de un curso de Educación Continua sobre Vacunación con abordaje a un instrumento didáctico resumido y actualizado sobre el Calendario Nacional de Vacunación del Ministerio de la Salud, adaptado por la autora de la investigación a la realidad local, con el objetivo de una mejor practica de esos profesionales servindo como una Orientación de la rutina para la acción cotidiana ejecutada en las salas de Vacunación. 
El Objetivo General de la investigación es: desarrolhar un Curso de Educación Continua sobre el Calendário Nacional de Vacunación para el Equipo de Enfermería en el municipio de Santa María do Pará, en el estado de Pará, Brasil. Los Objetivos Específicos son: 1) Evaluar un instrumento didáctico actualizado sobre el Calendario Nacional de Vacunación para facilitar la práctica profesional del Equipo de Enfermería; 2) Identificar las competencias y habilidades del Equipo de Enfermería propias en sala de vacunación.

2.-Programa Nacional de Inmunización (PNI): El Calendario Nacional de Vacunación.

Feliciano (2002), refiere que "La erradicación mundial de la viruela trae la esperanza de la erradicación de otras enfermedades evitables por la vacunación. Así, en 1974, la OMS creó el Programa Alargado de Vacunación, generalmente conocido por la designación anglosajona "Expanded Programme of Immunization" (EPI), el cual incluye seis vacunas: Tuberculosis, Difteria, Tétano, Tos convulsa, Poliomielitis y Sarampión, más tarde adicionaría la Fiebre-Amarilla y la Hepatitis B".

Figueiredo (2011), resalta que el "Programa Ampliado de Inmunización (PAI) fue creado en los años de 1970 por la Organización Mundial de la Salud, con el objetivo de reducir la Mortalidad y Morbididad Infantil de seis enfermedades prevenibles por la inmunización: Sarampión, Coqueluche, Poliomielitis, Tuberculosis, Tétano, Difteria, mediante la aplicación de las vacunas correspondientes. Basado en este programa, Brasil implantó el Programa Nacional de Inmunización (PNI), fue creado por el Ministerio de la Salud en septiembre 1973 e institucionalizado por el decreto $\mathrm{n}^{0}$ 78.231 de 12 de agosto de 1976. Tiene por objetivo promover el control de las enfermedades inmunoprevenibles por medio de estabelecimiento de normas y parámetros técnicos para la utilización de Inmunobiologicos, buscando coordinar las acciones de inmunización desarrolladas en la red de servicios de salud brasileña utilizados en el país (David \& Alexandre, 2011).

Para este autor el Calendario de Vacunación de Inmunización, es aquel definido por el Programa Nacional de Inmunizaciones del Ministerio de la Salud (PNI/MS) y corresponde al conjunto de vacunas consideradas de interés prioritario a la Salud Pública del país. Existen vacunas recomendadas desde el nacimiento hasta a tercera edad y distribuidas gratuitamente en los centros de Vacunación de la red pública.

De ese modo la creación del PNI Brasil por el Ministerio de Salud. Portaría MS $n^{0} 78.231 /$ GM/MS - 12 de agosto de 1976. Reglamenta la Ley $n^{0} 6.259$, del 30 de octubre de 1975, que dispone sobre la organización de las acciones de Vigilancia Epidemiológica, sobre el Programa Nacional de Inmunizaciones, establece normas relativas a notificación compulsoria de enfermedades y otras providencias de Vacunación. Por lo tanto, fue incorporada en la rutina de los servicios de salud en rol de enfermedades contempladas con esa medida de prevención.

Actualmente, el Calendario Nacional de Vacunación contempla: Calendario Básico de los Niños, Calendario del Adolescentes, Adultos, Gestantes y Ancianos, como 
muestra la Fuente del Ministerio de Salud de 2014. Además de esas, también está disponible la vacuna para la Rabia (inactivada), obtenida en cultura de células y los sueros heterólogos antitetánico, antidiftérico y antirrábico. También pueden ser puestos en disposición, frente a riesgos específicos, sueros antiofídicos y anti aracnídeos.

En la evolución del PNI, nuevas necesidades fueron contempladas, como es el caso, de la inmunización de personas con daños a la salud que comprometen su sistema inmunológico. Por esa y otras necesidades, el PNI implantó, a partir de 1993, los Centros de Referencia para Inmunobiologicos Especiales - CRIE y hoy existe este servicio en cada Estado y en el Distrito Federal.

David \& Alexandre (2011), cita que la Red de Frio es el proceso de recibimiento, almacenamiento, conservación, manipulación, distribución y transporte de los Inmunobiologicos del Programa Nacional de Inmunizaciones (PNI), con el objetivo de mantener la temperatura adecuada de estos productos, desde el laboratorio productor hasta el momento de su utilización, garantizando así sus características iniciales para obtener inmunidad.

Considerando por la Portaria № 1.498, de 19 de Julio de 2013: Art. $1^{\circ}$ Esta Portaria redefine el Calendario Nacional de Vacunación, el Calendario Nacional de Vacunación de los Pueblos Indígenas y las Campañas Nacionales de Vacunación, en el ámbito del Programa Nacional de Inmunizaciones (PNI), en todo el território nacional. Art. $2^{\circ}$ Los Calendarios y las Campañas Nacionales de Vacunación tienen por objetivo y control, la eliminación y la erradicación de enfermedades inmunoprevenibles. Art. $5^{\circ}$ El Ministerio de Salud será responsable por la adquisición y por el abastecimiento a las Secretarias de Salud de los Estados, del Distrito Federal y de los Municipios, de las vacunas recomendadas en los Calendarios y en las Campañas Nacionales de Vacunación. Art. $6^{\circ}$ Compete a las Secretarias de Salud de los Estados, del Distrito Federal y de los Municipios la gestión de la Red de Frio.

Párrafo único. Se considera Red de Frio el proceso de almacenamiento, conservación, manoseo, distribución y transporte de Imunobiológicos, siendo organizada por Equipo Técnico calificado para ejecución de sus actividades; equipamientos y procedimientos estandarizados para mantener las condiciones adecuadas de refrigeración y las características de los Imunobiológicos, desde el laboratorio productor hasta el momento de su administración.

Art. $7^{\circ}$ Los registro de las informaciones en cuanto a las vacunas administradas será hecho en los instrumentos estandarizados en el ámbito del PNI, obedeciendo al flujo y la periodicidad allí definidos, siendo responsabilidad de las Secretarias de Salud del Distrito Federal y de los Municipios, en lo que se refiere al registro en el Sistema de Información del Programa Nacional de Inmunizaciones (SIPNI); Art. $8^{\circ} \mathrm{La}$ comprobación de la Vacunación será hecha por medio del cartón o carnet de Vacunación, emitido por las unidades de Salud Pública y/o privada, debidamente autorizadas en el ámbito del S.U.S, conteniendo las siguientes informaciones: 
nombre de la vacuna; fecha; número del lote; laboratorio productor; unidad vacunadora; nombre del vacunador.

Haciendo una comparación con algunos países latinoamericanos elegimos las Recomendaciones Nacionales de Vacunación de Argentina 2012, donde se destaca el crecimiento del Calendario Nacional de Vacunación en este período, siendo uno de los más completos y modernos de Latinoamérica. La incorporación de nuevas vacunas seguras y eficaces en forma gratuita y obligatoria es uno de los indicadores de inclusión social y equidad más robustos que puede tener un país ya que facilita el acceso a esta herramienta clave de prevención para todos los sectores de la población. Por otro lado, la jerarquización de las vacunas que están incluidas en el calendario regular desde hace años y que han sido pilares fundamentales para la eliminación de enfermedades como la Poliomielitis, el Sarampión, la Rubéola y el Síndrome de Rubéola Congénita, es clave para sostener estos logros.

\section{3.-Educación en Salud: Educación Permanente, Educación Continua.}

Para Morosoni \& Col (2009), el potencial de la educación como proceso emancipatorio, en la interface con los movimientos sociales, tienen la categoría de práctica social, creadora/transformadora de la realidad, un aspecto central que está presente en las tesis que premian el pensamiento de Paulo Freire. Ese pensador ejerció fuerte influencia en el Movimiento de la Educación Popular en Salud, en América Latina y, particularmente, en Brasil. En la interface de la Educación y de la Salud, constituida con base en el pensamiento crítico sobre la realidad, se torna posible pensar en Educación en Salud como forma del hombre reunir y disponer recursos para actuar y transformar las condiciones objetivas, para lograr la salud como un derecho socialmente conquistado, a partir de la actuación individual y colectiva de sujetos político-sociales.

En esa visión comprendiendo la potencialidad educativa de los varios trabajadores de la salud (Médicos, Enfermeros, Nutricionistas, Psicólogos, Técnicos de Enfermería, Agentes Comunitarios de Salud, etc.) como educadores en salud interactuando con la población atendida con orientaciones de cuidados en salud. Ceccin \& Ferla (2009), refiere que la "Educación Permanente en Salud precisa ser entendida al mismo tiempo, como una práctica de enseñanza-aprendizaje y como una política de Educación en la Salud. Ella se parece con muchas vertientes brasileñas de la educación popular en salud y comparte muchos de sus conceptos, mientras que la Educación Popular tiene en vista la ciudadanía y la Educación Permanente tiene en vista el trabajo". Estos autores se fundamentan en el concepto de enseñanza problematizado (insertados de manera crítica en la realidad y sin superioridad del educador en relación al educando), de aprendizaje significativa (interesada en las experiencias anteriores y en las vivencias personales de los alumnos, desafiante del desear aprender más), o sea, enseñanza-aprendizaje basado en la producción de conocimientos.

En ese contexto Brasil, Ministerio de la Salud (2005), en su libro La Educación Permanente entra en la Rueda. Polos de educación permanente en salud. Conceptos 
y caminos a recorrer: donde aborda la Educación Permanente como aprendizaje hacia el trabajo, o sea, ella ocurre en el trabajo cotidiano de los profesionales de salud y trabaja con los problemas enfrentados en la realidad y un aprendizaje llevando en consideración las experiencias de los trabajadores que ya poseen una larga vida profesional. Podemos observar que los autores enfatizan la importancia de la Educación Permanente debido a los cambios tecnológicos y los nuevos conocimientos, movilizan las posibilidades y los saberes de los profesionales mostrando la necesidad de la continuidad en su formación.

Basado en este enfoque este autor de Brasil, Ministerio de la Salud (2005), en su libro La Educación Permanente entra en la Rueda. Polos de educación permanente en salud. Conceptos y Caminos a recorrer, donde relata que la Educación Continua es un proceso de adquisición secuencial y acumulativa de informaciones técnicocientíficas para el trabajador, por medio de Cursos de formación llevando en consideración las vivencias y experiencias laborales y de participación en el ámbito institucional 0 afuera de él.

Corroborando con este pensamiento, la Política Nacional de Atención Básica (PNAB, 2012), afirma la consolidación y el mejoramiento de la Atención Básica como importante reordenamiento del modelo de atención a la salud en Brasil, requieren un saber y un hacer en Educación Permanente y Educación Continua en los servicios de salud que estén incorporados a la teoría relacionada a la práctica profesional, adoptando con eso una postura transformadora de cambio en el comportamiento de los profesionales de la salud, obteniendo calidad en los servicios prestados y la satisfacción del usuario.

4.-La importancia de la Formación Continua para el Equipo de Enfermería en las Salas de Vacunación como Contribución en la Atención Primaria a la Salud (APS).

Para Souto (2000), la formación continua es un proceso que implica transformaciones, integraciones que tiene que ver con las personas en conjunto, incluyen lo cognitivo, en conjunto más amplio, que se refiere a la idea de experiencia con los otros (co-experiencia). Según Braga \& Melleiro (2009), en la enfermería, la responsabilidad de actualizar y de capacitar los profesionales está ligada al Servicio de Educación Continua (SEC), que debe preocuparse con las características de aprendizaje en cuanto un proceso dinámico, continuo, global, personal, gradual y acumulativo.

En esa dimensión, el objeto de esta investigación fue basada en la importancia de la Formación Continua sobre los estudios de Freire (1979 e 1983) ; en sus teorías de Educación Transformadora, considera la relación educador-educando, donde ambos aprenden, y en esta lógica, el enfermero sea el facilitador del proceso enseñanzaaprendizaje, utilizando una metodología problematizadora y transformadora estimulando el educando a tornarse un agente de cambio, que interactúa con todo el Equipo de Enfermería mediante las estrategias para realizar cursos de formación con actualizaciones y mejoras, estimulando la integración y desenvolvimiento de esos 
profesionales para mejorar la calidad de la atención a la población en las salas de vacunación en el municipio de Santa María do Pará.

De ese modo, el curso de Educación Continua es de fundamental importancia porque trabaja con dinámicas y elaboración creativa de un Instrumento Didáctico actualizado sobre el Calendario Nacional de Vacunación facilitando la práctica profesional del Equipo de Enfermería y con eso mejorar las competencias y habilidades técnicas en las salas de vacunación. Para Souza (2010), la Atención Primaria de la Salud (A.P.S), contempla un sistema de servicios de salud donde se ofrece la prestación de asistencia a la salud de los individuos, su acompañamiento continuo, a su familia, independiente de la ausencia o presencia de enfermedad garantizando un cuidado integral.

De acuerdo con el Manual de Normas y Procedimientos del Ministerio de Salud (2014), la sala de Vacunación es clasificada como área semicrítica. Debe ser destinada exclusivamente a la administración de los Inmunobiologicos, debiéndose considerar los diversos Calendarios Nacional de Vacunación existentes, por lo tanto en la sala de Vacunación es importante que todos los procedimientos desenvueltos promocionen la máxima seguridad, reduciendo el riesgo de contaminación para los individuos vacunados y también para el equipo de Vacunación.

El autor refuerza que las actividades de la sala de Vacunación son desarrolladas por el Equipo de Enfermería entrenado y capacitado para los procedimientos de manoseó, conservación, preparación y administración, registro y descarte de los residuos resultantes de las acciones de Vacunación. Es importante decir, que el Equipo de Enfermería es formado por el Enfermero y por el Técnico de Enfermería, siendo ideal la presencia de los vacunadores para realizar los procedimientos de administración de Vacunación, este equipo participa aún de la ciencia de la situación epidemiológica de la área de actuación en la cual la Vacunación está insertada, para el estabelecimiento de prioridades, la asignación de recursos y la orientación programática, cuando sea necesario.

Constatase que el Enfermero es responsable por la supervisión, monitoreo del trabajo del Técnico de Enfermería en la sala de Vacunación y por el proceso de Educación Permanente, Formación Continua y Educación Continua del Equipo de Enfermería, sin embargo para que este profesional de salud de nivel superior ejerza esa función con eficiencia, él también tiene que ser Capacitado por la gestión de la Atención Primaria en Salud en asociación con la Vigilancia Epidemiológica e Inmunización.

\section{5.-Metodologia.}

E Diseño del estudio es descriptivo y analítico: este corte prospectivo visó acompañar a los profesionales de la salud del Equipo de Enfermería de la ciudad de Santa María do Pará, PA, Brasil. La investigación se realizó durante el año 2014, de Enero hasta el mes de Julio, se trabajó en el proyecto buscando referencias 
bibliográficas, diseñando el Programa de Educación Continua, analizando la encuesta a ser aplicada y solicitando todos los aspectos éticos personales, a través de un Consentimiento Informado a las autoridades competentes, Centros de Salud y Coordinaciones en la ciudad de Santa María do Pará. Desde el mes de agosto hasta diciembre se realizaron los trabajos de Campo, completando el proceso de investigación. Se utilizó un cuestionario con preguntas abiertas y cerradas a los participantes de la investigación, antes y después de realizar el proceso de Educación Continua.

Los resultados de esta investigación se obtuvieron a través de la interpretación y análisis de las técnicas e instrumentos cuantitativos y cualitativos, porque permite llegar a los resultados y relatar los procedimientos del estudio de acuerdo con la perspectiva teórico-metodológica. De esa forma, será posible concretizar el estudio, permitiendo con más seguridad evaluar las afirmaciones que son hechas dentro del contexto de la investigación. Los resultados fueron procesados en planilla Excel e IBM SPSS STATISTICS 18, debidamente comprobadas por análisis estadísticos.

La población y muestra: El universo de la investigación está integrado por 28 participantes del Equipo de Enfermería compuesto por 10 Enfermeros y 18 Técnicos de Enfermería que trabajan con el Calendario Nacional, en las salas de Vacunación de las Unidades de Atención Primaria de Salud. La investigación de campo fue realizada en las salas de vacunación con el Equipo de Enfermería (técnicos y enfermeros), destacándose que todos estos profesionales de la salud son del cuadro funcional de la Secretaria Municipal de Salud de Santa María do Pará (SEMUS) y su local de trabajo está inserido en las Unidades de Salud de este municipio.

Criterios de Inclusión de la Investigación: son incluidos en la investigación los Enfermeros, Técnicos de Enfermería que actúan en Unidades de Atención Primaria de Salud con el Calendario Nacional de Vacunación en Santa María de Pará, estos deben estar trabajando en el período de la recolección de datos y presentar condiciones cognitivas y psicológicas para responder al cuestionario y firmar el consentimiento informado. Criterios de Exclusión de la Investigación: serán excluidos los Enfermeros, Técnicos de Enfermería que están alejados del trabajo por enfermedad, accidente, licencia por maternidad, o vacaciones en el período de la recolecta de datos y los casos que recusasen de participar de la investigación, que no firmaron el Termino del Consentimiento Libre e Informado T.C.L.I.

Cobertura de la Atención Primaria en el Municipio: El municipio posee 65 Agentes Comunitarios de Salud, que corresponden, a la cobertura de $94,20 \%$ de la población, 06 Equipos de Salud de la Familia, con cobertura del $60 \%$ de la población y 03 Equipos de Salud Bucal. Los aspectos éticos: la investigación fue realizada de acuerdo con las normas del Consejo Nacional de Salud, de acuerdo con la Resolución 466/12, los datos fueron colectados después de la aprobación previa del Comité de ética del Hospital Ophir Loiola (Plataforma Brasil CAAE: 36927213.6.0000.5550) (ANEXO I) y firma del Consentimiento Libre e Informado de los participantes (ANEXO VI). 


\section{6.- Discusión de los Resultados.}

En el presente capitulo se analizarán y discutirán los resultados obtenidos de los cuestionarios aplicados a los participantes de la investigación antes, durante y después de ofrecer el Curso de Educación Continua para el Equipo de Enfermería, sobre Calendario Nacional de Vacunación, municipio Santa María do Pará, Pará, Brasil 2014. Los fines prácticos seguirán el orden según se enunciaron los objetivos.

Gráfico 1. Edad de los participantes de la investigación sobre Educación Continua para el Equipo de Enfermería sobre el Calendario Nacional de Vacunación realizado en el municipio de Santa María do Pará en 2014.

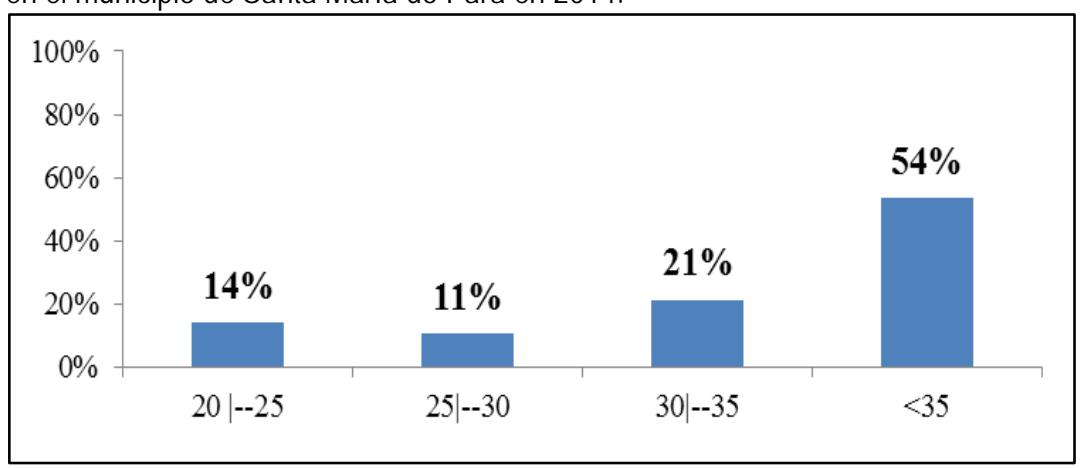

Fuente: La propia investigación (2018).

Participantes de la investigación: se observa que la mayoría (54\%), posee edad mayor de 35 años, seguida del intervalo entre 30 y 35 años (21\%). Para Lima et al (2009), en estudios brasileños sobre porcentaje de edad en profesionales de Equipos de Enfermería, se aproximan con el presente estudio teniendo los entrevistados una media de Edad de 30 años. En nuestra investigación consideramos que la mayoría de los participantes se encuentran por encima de 35 años de Edad debido al concurso público realizado en el municipio hace aproximadamente 10 años y muchos de estos profesionales empezaron sus labores a los 25 años de Edad.

Figura 2. Sexo de los participantes de la investigación sobre Educación Continua para el Equipo de Enfermería sobre el Calendario Nacional de Vacunación realizado en el municipio de Santa María do Pará en 2014. 


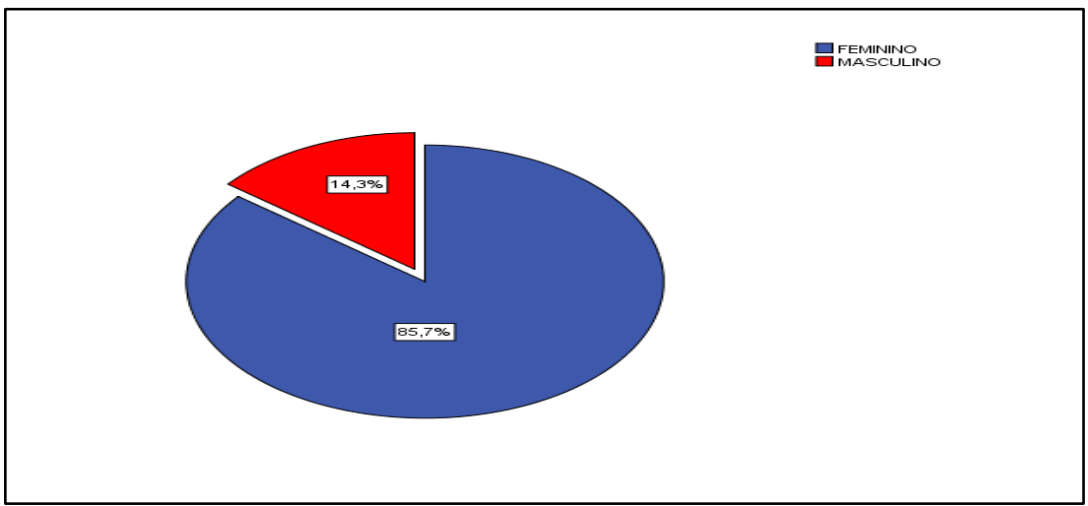

Fuente: La propia investigación (2018).

En la figura 2, se presenta el sexo de los participantes de la investigación, se nota que el mayor porcentaje es del sexo femenino $(85,7 \%)$ y los hombres corresponden $(14,3 \%)$. Corroborando con Machado et al (2012), que obtuvo un resultado similar en su investigación: Construyendo el Perfil de la Enfermería. Quedó evidenciado que la mayoría de los entrevistados (90\%) del Equipo de Enfermería es constituido por el Sexo Femenino, esto acontece por el proceso de feminización de la profesión que es formada casi integralmente de mujeres. Por otro lado, la Enfermería presenta un nuevo escenario con datos donde se observa un cambio creciente por la presencia masculina en la profesión, como una tendencia que vino para quedarse.

Figura 3. Profesión de los participantes de la investigación sobre Educación Continua para el Equipo de Enfermería sobre el Calendario Nacional de Vacunación realizado en el municipio de Santa María do Pará en 2014.

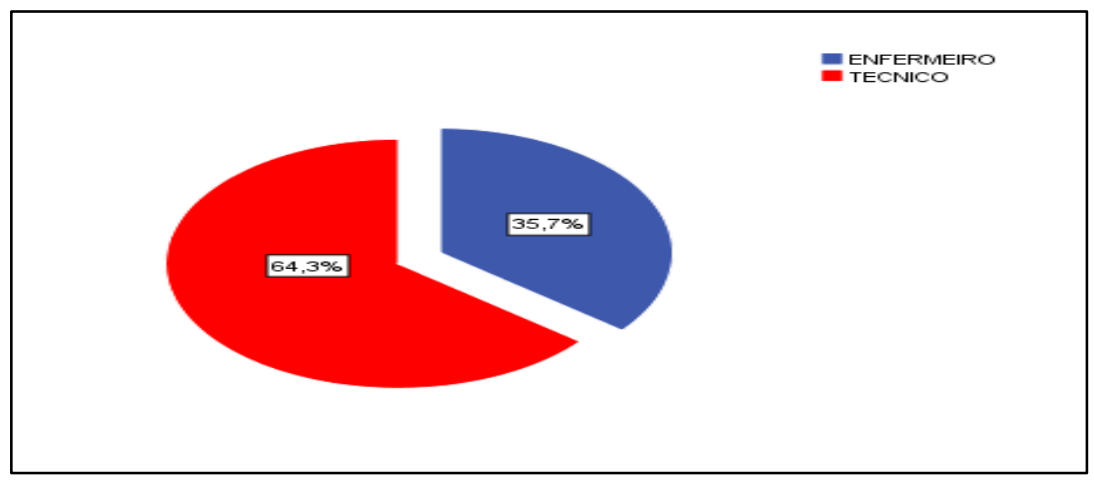

Fuente: La propia investigación (2018).

Sigue en la figura 3 la presentación del universo estudiado, se nota que ese está compuesto de profesionales del área de Enfermería, siendo ellos; Técnicos de Enfermería (64,3\%) y Enfermeros (35,7\%). Según Lima et al (2009), que detectó 78,87 \% de Técnicos de Enfermería en su investigación, esto es, evidenciado en el 
municipio de Santa María do Pará, que presenta en su cuadro funcional más Técnicos de Enfermería en la sala de vacunación que Enfermeros. Es notorio que todos los participantes (100\%) poseen la cartera de Vacunación actualizada.

Figura 4: La importancia del Curso de Educación Continua para el Equipo de Enfermería sobre el Calendario Nacional de Vacunación realizado en el municipio de Santa María do Pará en 2014.

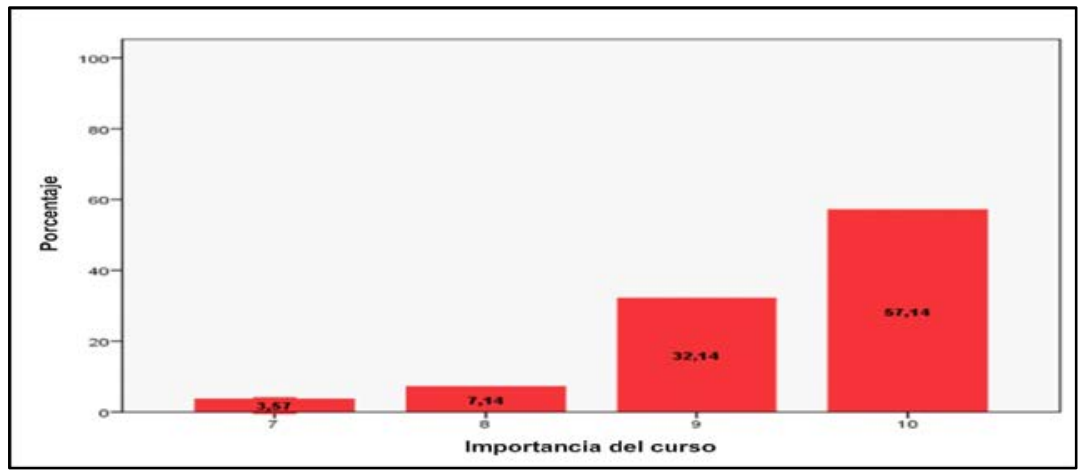

Fuente: La propia investigación (2018).

Un factor importante es la relación de la opinión de los participantes en cuanto a la importancia del curso de Educación Continua en relación a la actualización en vacunación para la actuación profesional. Para tal situación, se hizo necesaria una escala de 1 a 10 con el fin de cuantificar tales resultados. La figura 4, demuestran que dentro de la escala, se presentan apenas valores a partir de 7 , siendo que más de la mitad $(57,1 \%)$ de los profesionales evaluaron con criterios máximos la necesidad de recibir el curso en cuestión.

En consonancia sobre la importancia del Curso de Educación Continua en relación a la actualización de vacunación para la actuación profesional del Equipo de Enfermería. Lima et al (2009), verificaran que el 50\% de los participantes de la investigación consideran importante el curso, entrenamiento y mejora de las habilidades prácticas, con el objetivo de tornar el aprendizaje más significativo y contextualizado, debido a eso el Curso de Educación Continua es importante para ofrecer oportunidad de reciclar y actualizar al Equipo de Enfermería, mejorando sus conocimientos científicos estimulando cada vez más a adquirir competencias y habilidades prácticas en su actuación profesional en la sala de vacunación.

Figura 5. Conocimiento del Manejo de la Temperatura y organización de la Heladera para conservación de las vacunas, según los participantes del Curso de Educación Continua para el Equipo de Enfermería sobre el Calendario Nacional de Vacunación realizado en el municipio de Santa María do Pará en 2014. 


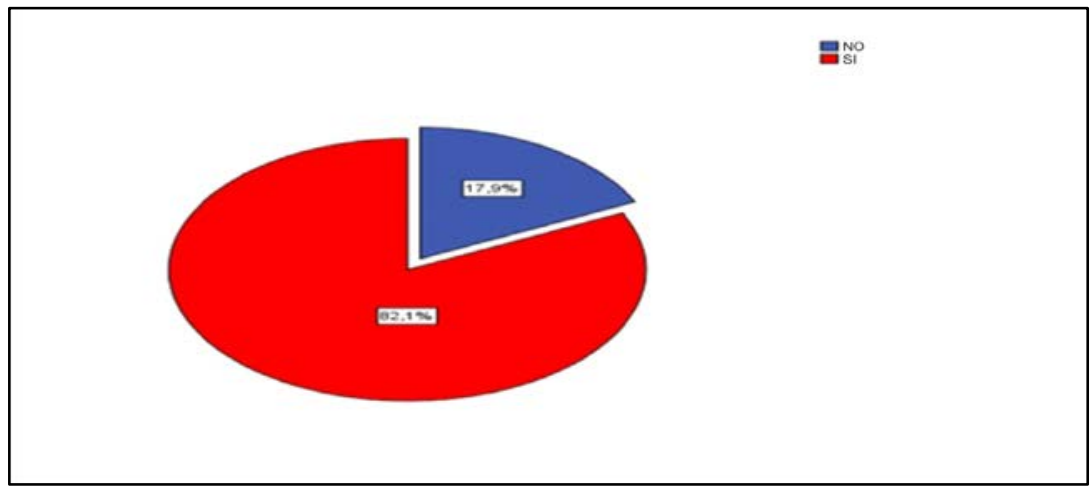

Fuente: La propia investigación (2018).

La Figura 5: Una vez terminado el Curso de Educación Continua, cuando se preguntó a los participantes acerca de la contribución en relación a la conservación de Inmunobiologicos en las salas de vacunas de las unidades de salud, 82,1\% respondieron afirmativamente. Un análisis se hace necesario para conocer si los participantes saben constatar la temperatura de la heladera con termómetros digitales y de cabo extensor, además de organizar la heladera de conservación de vacunas de acuerdo con las normas establecidas por el Ministerio de Salud. Basándose en tal situación, se verifica que el $82,1 \%$ saben realizar tales procedimientos, mientras que el $17,9 \%$ respondió negativamente.

Este autor enfatiza que en la sala de Vacunación las actividades deben ser desarrolladas por un Equipo de Enfermería entrenado para la manipulación, conservación y administración de los Inmunobiológicos. Ese Equipo debe estar compuesto, preferentemente, por uno o dos Técnicos de Enfermería, contando con la participación de un Enfermero responsable por la supervisión y entrenamiento en el servicio. Para tal supervisión se le exige al Enfermero la Responsabilidad Técnica (RT) por el servicio, que está establecido en la Resolución № 302 de 2005 del Consejo Federal de Enfermería.

\section{7.-Conclusiones.}

A partir de técnicas de análisis de datos descriptivos, concluimos que los objetivos de la investigación fueron logrados. Se desarrolló un Curso de Educación Continua sobre el Calendario Nacional de Vacunación para el Equipo de Enfermería, que han participado evaluando muy importante la actividad educativa para actualizar sus conocimientos en el 57 \% y por eso los cursos de Educación Continua son importantes para ofrecer oportunidades de reciclar y actualizar conocimientos cientifico para el Equipo de Enfermería, mejorando, estimulando cada vez más a adquirir Competencias y Habilidades prácticas en su actuación profesional en las Salas de Vacunación. 
Se elaboró un Instrumento Didáctico donde se incluyó la última actualización del Calendario Nacional de Vacunación como parte del Curso de Educación Continua, el cual es considerado por el 96,4 \%, de los participantes como importante para alcanzar las Metas del Ministerio de la Salud en relación a las Campañas Nacionales de Vacunación y así facilitar la práctica profesional.

Se observó que los participantes del Curso de Educación Continua adquirieron Habilidades y Competencias en relación a la actualización de vacunas para el Equipo de Enfermería y los principales conocimientos (teórico-prácticos) adquiridos en el curso de mejoramiento. Se evidencian las principales Habilidades y Competencias adquiridas por Equipo de Enferméria durante el Curso de Educación Continua: Esquema de vacunación (32,1\%), Efectos Adversos $(21,4 \%)$ y organización de una sala de vacunación ideal $(14,3 \%)$.

\section{8.-Bibliografia.}

Bernadete, A.L., Rosana, D. (2011). Vacunas: Orientações Práticas. Son Paulo. Martinari. Resolución Ministerial $n^{\circ} 2162$ Presidencia de la Nación. Ministerio de Salud Buenos Aires: ProNaCEl.

Braga, A. T.; Melleiro, M. M.(2009). Percepção de la equipe de enfermería acerca de um serviço de educação continuada. Revista Escola Enfermagen. V.2, n.43, São Paulo: Universidade São Paulo. Escola Enfermería. p. 1216-1220.

Cavalcante, C. (2008). Vacunación e Biossegurança: el olhar de los profesionales de enfermería. Dissertação de Mestrado, Universidade Federal del Rio Grande del Norte. Ceccin, R. B.; Ferla, A. A. (2009). Educação Permanente em Salud. Disponível em: http://www.epsjv.fiocruz.br/dicionario/verbetes/edusau.html acesso em: 16 novembro de 2013. XXX

Feliciano, J. (2002). La vacuna e la sua História. Cadernos de la Dirección geral de la Salud. (2). 3-7.

Figueiredo, G.L.L.A., Pina, J.C. Tonete V.L.P, Lima. (2011). Experiencias de família sem la Inmunización de niños brasileños menores de los años. Argentina: Latino-Am.

Freire, P. (1979). Educação como prática de la liberdade. 17.ed. Rio de Janeiro: Paz e Terra. (2015). Recuperado de: http://www.monografias.com/paulofreire/paulofreire.

Freire, P. (1983). Pedagogia do Oprimido. 13a . ed. Rio de Janeiro, Paz e Terra. (Coleção el Mundo, hoy, v.21). Recuperado de: http://www.monografias.com/paulofreire/paulofreire.

Marconi, M.D.L.A, Lakatos, E.M. (2003). Fundamentos de Metodologia Cientifica. Son Paulo: Atlas. 
Morosini, M.V., Fonseca, L.A.F., Pereira, I.B. (2009). Educação em Salud. Recuperado de: http://www.epsjv.fiocruz.br/dicionario/verbetes/edusau.html.

Portaria $n^{0}$ 1.498. (2013). Redefine el Calendário Nacional de Vacunación, el Calendário Nacional de Vacunación de los Povos Indígenas e as Campanhas Nacionais de Vacunación, ENEL âmbito del Programa Nacional de Inmunizaciones (PNI), em todo el território nacional. Ministério de Salud. Recuperado de: http://bvsms.saude.gov.br/bvs/saudelegis/gm.

Sampieri, H.R. (2006). Metodología de la Investigación. México: Mc Graw- Hill Interamericano.

Souto, M. (2000). Grupos y dispositivos de formación. Formación de formadores. Carrera de Especialización de Posgrado. Son Paulo: Los documentos.

Souza, S.K. (2010). Análise de Cobertura De vacunación e de la Estratégia. Campanhista en el Município de Teófilo Antônio Minas Gerais en el período de 2005 la 2009. Belo Horizonte: Universidade Federal de Minas Gerais.

Teixeira, E. (2013). As Três Metodologias: Academia, Ciência, e la Pesquisa. Rio de Janeiro: Petrópolis. 